Honam Mathematical J. 36 (2014), No. 3, pp. 531-542

http://dx.doi.org/10.5831/HMJ.2014.36.3.531

\title{
THE INCOMPLETE LAURICELLA AND FIRST APPELL FUNCTIONS AND ASSOCIATED PROPERTIES
}

\author{
Junesang Choi*, Rakesh K. Parmar, and Purnima Chopra
}

\begin{abstract}
Recently, Srivastava et al. [18] introduced the incomplete Pochhammer symbol and studied some fundamental properties and characteristics of a family of potentially useful incomplete hypergeometric functions. Here we introduce the incomplete Lauricella function $\gamma_{D}^{(n)}$ and $\Gamma_{D}^{(n)}$ of $n$ variables, and investigate certain properties of the incomplete Lauricella functions, for example, their various integral representations, differential formula and recurrence relation, in a rather systematic manner. Some interesting special cases of our main results are also considered.
\end{abstract}

\section{Introduction, definitions and preliminaries}

Throughout this paper, $\mathbb{N}, \mathbb{Z}^{-}$, and $\mathbb{C}$ denote the sets of positive integers, negative integers, complex numbers, respectively, $\mathbb{N}_{0}:=\mathbb{N} \cup\{0\}$ and $\mathbb{Z}_{0}^{-}:=\mathbb{Z}^{-} \cup\{0\}$. by

The familiar incomplete Gamma functions $\gamma(s, x)$ and $\Gamma(s, x)$ defined

$$
\gamma(s, x):=\int_{0}^{x} t^{s-1} e^{-t} d t \quad(\Re(s)>0 ; x \geqq 0)
$$

and

$$
\Gamma(s, x):=\int_{x}^{\infty} t^{s-1} e^{-t} d t \quad(x \geqq 0 ; \Re(s)>0 \text { when } x=0)
$$

Received May 19, 2014. Accepted July 7, 2014.

2010 Mathematics Subject Classification. Primary 33B15, 33B20, 33C05, 33C15, 33C20; Secondary 33B99, 33C99, $60 \mathrm{~B} 99$.

Key words and phrases. Gamma functions, incomplete gamma function, Pochhammer symbol, incomplete Pochhammer symbol, incomplete generalized hypergeometric functions, Lauricella functions, Appell function, Bessel and modified Bessel functions, incomplete first Appell function, incomplete Lauricella function of $n$ variables.

${ }^{*}$ Corresponding author 
respectively, satisfy the following decomposition formula:

$$
\gamma(s, x)+\Gamma(s, x):=\Gamma(s) \quad(\Re(s)>0),
$$

where $\Gamma(s)$ is the familiar Gamma function defined by

$$
\Gamma(s)=\int_{0}^{\infty} t^{s-1} e^{-t} d t \quad(\Re(s)>0) .
$$

Each of these functions plays an important role in the study of the analytic solutions of a variety of problems in diverse areas of science and engineering (see, e.g., $[1,2,6,7,9,11,12,13,19,20,21,23,24]$ ).

Very recently, Srivastava et al. [18] introduced and studied in a rather systematic manner the following two families of generalized incomplete hypergeometric functions:

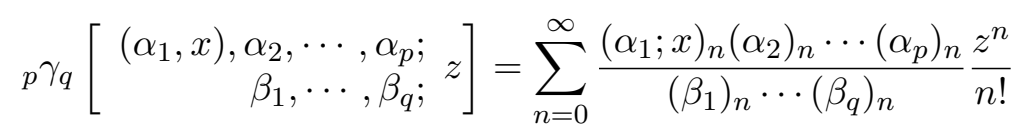

and

$$
{ }_{p} \Gamma_{q}\left[\begin{array}{r}
\left(\alpha_{1}, x\right), \alpha_{2}, \cdots, \alpha_{p} ; z \\
\beta_{1}, \cdots, \beta_{q} ;
\end{array}\right]=\sum_{n=0}^{\infty} \frac{\left[\alpha_{1} ; x\right]_{n}\left(\alpha_{2}\right)_{n} \cdots\left(\alpha_{p}\right)_{n}}{\left(\beta_{1}\right)_{n} \cdots\left(\beta_{q}\right)_{n}} \frac{z^{n}}{n !}
$$

where, in terms of the incomplete Gamma functions $\gamma(s, x)$ and $\Gamma(s, x)$ defined by (1.1) and (1.2), the incomplete Pochhammer symbols $(\lambda ; x)_{\nu}$ and $[\lambda ; x]_{\nu}(\lambda, \nu \in \mathbb{C} ; x \geqq 0)$ are defined as follows:

$$
(\lambda ; x)_{\nu}:=\frac{\gamma(\lambda+\nu, x)}{\Gamma(\lambda)} \quad(\lambda, \nu \in \mathbb{C} ; x \geqq 0)
$$

and

$$
[\lambda ; x]_{\nu}:=\frac{\Gamma(\lambda+\nu, x)}{\Gamma(\lambda)} \quad(\lambda, \nu \in \mathbb{C} ; x \geqq 0)
$$

so that, obviously, these incomplete Pochhammer symbols $(\lambda ; x)_{\nu}$ and $[\lambda ; x]_{\nu}$ satisfy the following decomposition relation:

$$
(\lambda ; x)_{\nu}+[\lambda ; x]_{\nu}:=(\lambda)_{\nu} \quad(\lambda, \nu \in \mathbb{C} ; x \geqq 0),
$$

where $(\lambda)_{n}$ is the Pochhammer symbol defined (for $\lambda \in \mathbb{C}$ ) by (see $[20$, p. 2 and p. 5]):

$$
\begin{aligned}
(\lambda)_{n}: & = \begin{cases}1 & (n=0) \\
\lambda(\lambda+1) \ldots(\lambda+n-1) & (n \in \mathbb{N})\end{cases} \\
& =\frac{\Gamma(\lambda+n)}{\Gamma(\lambda)} \quad\left(\lambda \in \mathbb{C} \backslash \mathbb{Z}_{0}^{-}\right) .
\end{aligned}
$$


As already pointed out by Srivastava et al. [18, Remark 7], since

$$
\left|(\lambda ; x)_{n}\right| \leqq\left|(\lambda)_{n}\right| \quad \text { and } \quad\left|[\lambda ; x]_{n}\right| \leqq\left|(\lambda)_{n}\right| \quad\left(\lambda \in \mathbb{C} ; n \in \mathbb{N}_{0} ; x \geqq 0\right)
$$

the precise sufficient conditions under which the infinite series in definitions (1.5) and (1.6) would converge absolutely can be derived from those that are well-documented in the case of the generalized hypergeometric function ${ }_{p} F_{q}\left(p, q \in \mathbb{N}_{0}\right)$ (see [15, p. 72-73] and [22, p. 20]); see also, $[3,4,10]$ and [17]). Indeed, in their special case when $x=0$, both ${ }_{p} \gamma_{q}\left(p, q \in \mathbb{N}_{0}\right)$ and ${ }_{p} \Gamma_{q}\left(p, q \in \mathbb{N}_{0}\right)$ would reduce immediately to the extensively-investigated generalized hypergeometric function ${ }_{p} F_{q}(p, q \in$ $\left.\mathbb{N}_{0}\right)$.

Furthermore, it is easy to see from the definitions (1.5) and (1.6), we have the following decomposition formula:

$$
\begin{aligned}
{ }_{p} \gamma_{q}\left[\begin{array}{r}
\left.\left(\alpha_{1}, x\right), \alpha_{2}, \ldots, \alpha_{p} ; z\right] \\
\beta_{1}, \ldots, \beta_{q} ; z
\end{array}\right]+{ }_{p} \Gamma_{q}\left[\begin{array}{r}
\left(\alpha_{1}, x\right), \alpha_{2}, \ldots, \alpha_{p} ; z \\
\beta_{1}, \ldots, \beta_{q} ; z
\end{array}\right] \\
\quad={ }_{p} F_{q}\left[\begin{array}{r}
\alpha_{1}, \alpha_{2}, \ldots, \alpha_{p} ; z \\
\beta_{1}, \ldots, \beta_{q} ;
\end{array}\right]
\end{aligned}
$$

in terms of the familiar generalized hypergeometric function ${ }_{p} F_{q}$.

Motivated essentially by the demonstrated potential for applications of these incomplete hypergeometric functions $p \gamma_{q}$ and ${ }_{p} \Gamma_{q}$, in many diverse areas of mathematical, physical, engineering and statistical sciences (see, for details, $[5,18]$ and the references cited therein), here, we aim at investigating, in a rather systematic manner, the incomplete family of Lauricella's functions $\gamma_{D}^{(n)}$ and $\Gamma_{D}^{(n)}$ of $n$ variables and investigate certain properties of the incomplete Lauricella functions, for example, their various integral representations, differential formula and recurrence relations. Some integral representations involving Bessel and modified Bessel functions are also presented. In particular, for $n=2$, we obtain similar results for the first Appell functions $\gamma_{1}$ and $\Gamma_{1}$.

\section{Incomplete Lauricella and first Appell functions}

In terms of the incomplete Pochhammer $\operatorname{symbol}(\lambda ; x)_{v}$ and $[\lambda ; x]_{v}$ defined by (1.7) and (1.8), we introduce the incomplete families of Lauricella hypergeometric functions $\gamma_{D}^{(n)}$ and $\Gamma_{D}^{(n)}$ of $n$ variables as follows: For $\alpha, \beta_{1}, \ldots, \beta_{n} \in \mathbb{C}$ and $\gamma_{1}, \ldots, \gamma_{n} \in \mathbb{C} \backslash \mathbb{Z}_{0}^{-}$, 


$$
\begin{aligned}
\gamma_{D}^{(n)} & {\left[(\alpha, x), \beta_{1}, \ldots, \beta_{n} ; \gamma ; x_{1}, \ldots, x_{n}\right] } \\
& =\sum_{m_{1}, \ldots, m_{n}=0}^{\infty} \frac{(\alpha ; x)_{m_{1}+\cdots+m_{n}}\left(\beta_{1}\right)_{m_{1}} \cdots\left(\beta_{n}\right)_{m_{n}}}{(\gamma)_{m_{1}+\cdots+m_{n}}} \frac{x_{1}^{m_{1}}}{m_{1} !} \cdots \frac{x_{n}^{m_{n}}}{m_{n} !}
\end{aligned}
$$

and

$$
\begin{aligned}
\Gamma_{D}^{(n)} & {\left[(\alpha, x), \beta_{1}, \ldots, \beta_{n} ; \gamma ; x_{1}, \ldots, x_{n}\right] } \\
& =\sum_{m_{1}, \ldots, m_{n}=0}^{\infty} \frac{[\alpha ; x]_{m_{1}+\cdots+m_{n}}\left(\beta_{1}\right)_{m_{1}} \cdots\left(\beta_{n}\right)_{m_{n}}}{(\gamma)_{m_{1}+\cdots+m_{n}}} \frac{x_{1}^{m_{1}}}{m_{1} !} \cdots \frac{x_{n}^{m_{n}}}{m_{n} !} .
\end{aligned}
$$

In view of (1.9), these incomplete families of Lauricella functions satisfy the following decomposition formula:

$$
\gamma_{D}^{(n)}+\Gamma_{D}^{(n)}=F_{D}^{(n)},
$$

where $F_{D}^{(n)}$ is the familiar Lauricella function (see [21, 22]).

Theorem 1. The incomplete Lauricella's function $\gamma_{D}^{(n)}$ and $\Gamma_{D}^{(n)}$ satisfy the following partial differential equation:

$$
\begin{aligned}
& x_{j}\left(1-x_{j}\right) \frac{\partial^{2} u}{\partial x_{j}^{2}}+\left(1-x_{j}\right) \sum_{k=1, k \neq j}^{n} x_{k} \frac{\partial^{2} u}{\partial x_{k} \partial x_{j}} \\
& \quad+\left[\gamma_{j}-\left(\alpha+\beta_{j}+1\right) x_{j}\right] \frac{\partial u}{\partial x_{j}}-\beta_{j} \sum_{k=1, k \neq j}^{n} x_{k} \frac{\partial u}{\partial x_{k}}-\alpha \beta_{j} u=0,
\end{aligned}
$$

where $j=1, \ldots, n$ and

$$
\begin{aligned}
u=u\left(x_{1}, \ldots, x_{n}\right):= & \gamma_{D}^{(n)}\left[(\alpha, x), \beta_{1}, \ldots, \beta_{n} ; \gamma ; x_{1} \ldots, x_{n}\right] \\
& +\Gamma_{D}^{(n)}\left[(\alpha, x), \beta_{1}, \ldots, \beta_{n} ; \gamma ; x_{1}, \ldots, x_{n}\right] .
\end{aligned}
$$

Proof. In the light of (2.3), it is easy to prove (2.4) since $F_{D}^{(n)}$ satisfies the same system of partial differential equations as in (2.4).

In particular, for $n=2$, the corresponding incomplete families of first Appell hypergeometric functions

$$
\gamma_{D}^{(2)}:=\gamma_{1} \quad \text { and } \quad \Gamma_{D}^{(2)}:=\Gamma_{1}
$$


are given by

$$
\begin{array}{r}
\gamma_{1}\left[(\alpha, x), \beta_{1}, \beta_{2} ; \gamma ; x_{1}, x_{2}\right]=\sum_{m, n=0}^{\infty} \frac{(\alpha ; x)_{m+n}\left(\beta_{1}\right)_{m}\left(\beta_{2}\right)_{n}}{(\gamma)_{m+n}} \frac{x_{1}^{m}}{m !} \frac{x_{2}^{n}}{n !} \\
=\sum_{m=0}^{\infty} \frac{(\alpha)_{m}\left(\beta_{1}\right)_{m}}{(\gamma)_{m}}{ }_{2} \gamma_{1}\left[\begin{array}{r}
(\alpha+m, x), \beta_{2} ; \\
\gamma+m ;
\end{array} x_{2}\right] \frac{x_{1}^{m}}{m !}
\end{array}
$$

and

$$
\begin{array}{r}
\Gamma_{1}\left[(\alpha, x), \beta_{1}, \beta_{2} ; \gamma ; x_{1}, x_{2}\right]=\sum_{m, n=0}^{\infty} \frac{[\alpha ; x]_{m+n}\left(\beta_{1}\right)_{m}\left(\beta_{2}\right)_{n}}{(\gamma)_{m+n}} \frac{x_{1}^{m}}{m !} \frac{x_{2}^{n}}{n !} \\
=\sum_{m=0}^{\infty} \frac{(\alpha)_{m}\left(\beta_{1}\right)_{m}}{(\gamma)_{m}}{ }_{2} \Gamma_{1}\left[\begin{array}{r}
(\alpha+m, x), \beta_{2} ; \\
\gamma+m ;
\end{array} x_{2}\right] \frac{x_{1}^{m}}{m !}
\end{array}
$$

respectively.

Remark 1. The special cases of (2.1) and (2.2) when $n=1$ are seen to yield the known incomplete families of Gauss hypergeometric functions [18]:

$$
\gamma_{D}^{(1)}={ }_{2} \gamma_{1}\left[(\alpha, x) ; \beta_{1} ; \gamma ; x_{1}\right] \text { and } \Gamma_{D}^{(1)}={ }_{2} \Gamma_{1}\left[(\alpha, x) ; \beta_{1} ; \gamma ; x_{1}\right],
$$

respectively.

It is noted in passing that, in view of the formula (2.3), it is sufficient to discuss the properties and characteristics of the incomplete Lauricella and first Appell functions $\Gamma_{D}^{(n)}$ and $\Gamma_{1}$.

\section{Integral Representations of Incomplete Lauricella and first Appell functions}

Here, we present certain integral representations of incomplete Lauricella function by applying (1.2) and (1.8). We also obtain some integral representations involving Bessel and modified Bessel functions. Further, we give corresponding results for incomplete first Appell functions for $n=2$.

Theorem 2. The following integral representation for incomplete Lauricella's function $\Gamma_{D}^{(n)}$ in (2.2) holds true: 


$$
\begin{aligned}
& \Gamma_{D}^{(n)} {\left[(\alpha, x), \beta_{1}, \ldots, \beta_{n} ; \gamma ; x_{1}, \ldots, x_{n}\right] } \\
&=\frac{1}{\Gamma(\alpha)} \int_{x}^{\infty} e^{-t} t^{\alpha-1} \Phi_{2}^{(n)}\left[\beta_{1}, \ldots, \beta_{n} ; \gamma ; x_{1} t, \ldots, x_{n} t\right] d t \\
&\left(x \geqq 0 ; \max \left\{\Re\left(x_{1}\right), \ldots, \Re\left(x_{n}\right)\right\}<1 ; \Re(\alpha)>0 \text { when } x=0\right),
\end{aligned}
$$

where $\Phi_{2}^{(n)}$ is one of the confluent forms of Lauricella series in $n$ variables defined by (see, e.g., [21, p. 34, Eq. (8)])

$$
\begin{aligned}
\Phi_{2}^{(n)} & {\left[b_{1}, \ldots, b_{n} ; c ; x_{1}, \ldots, x_{n}\right] } \\
& =\sum_{m_{1}, \ldots, m_{n}=0}^{\infty} \frac{\left(b_{1}\right)_{m_{1}} \cdots\left(b_{n}\right)_{m_{n}}}{(c)_{m_{1}+\cdots+m_{n}}} \frac{x_{1}{ }^{m_{1}}}{m_{1} !} \cdots \frac{x_{n}{ }^{m_{n}}}{m_{n} !} .
\end{aligned}
$$

Proof. Using the definition of the incomplete Pochhammer symbol $[\alpha ; x]_{m_{1}+\cdots+m_{n}}$ in (2.2) by considering the integral representation from (1.2) and (1.8) and using the definition [21, p. 34(8)], we are led to the desired result.

Theorem 3. The following n-multiple integral representation for $\Gamma_{D}^{(n)}$ in $(2.2)$ holds true:

$$
\begin{aligned}
\Gamma_{D}^{(n)}\left[(\alpha, x), \beta_{1}, \ldots, \beta_{n} ; \gamma ; x_{1}, \ldots, x_{n}\right] \\
=\frac{1}{\Gamma\left(\beta_{1}\right) \cdots \Gamma\left(\beta_{n}\right)} \int_{0}^{\infty} \cdots \int_{0}^{\infty} e^{-s_{1}-\ldots-s_{n}} s_{1}^{\beta_{1}-1} \cdots s_{n}^{\beta_{n}-1} \\
\quad \times{ }_{1} \Gamma_{1}\left((\alpha, x) ; \gamma ; x_{1} s_{1}+\cdots+x_{n} s_{n}\right) d s_{1} \cdots d s_{n} \\
\left(x \geqq 0 ; \Re\left(\beta_{1}\right), \ldots, \Re\left(\beta_{n}\right)>0 \text { when } x=0\right) .
\end{aligned}
$$

Proof. Using the integral representation of the classical Pochhammer symbol for $(\beta)_{m_{1}}, \cdots,(\beta)_{m_{n}}$ in the definition (2.2) and using the definition of incomplete confluent hypergeometric function for the case $p=1$ and $q=1$ in [18, p. 675, Eq.(4.1)], we are led to the desired result.

Theorem 4. The following $n+1$-multiple integral representation for $\Gamma_{D}^{(n)}$ in $(2.2)$ holds true: 


$$
\begin{aligned}
\Gamma_{D}^{(n)}\left[(\alpha, x), \beta_{1}, \ldots, \beta_{n} ; \gamma ; x_{1}, \ldots, x_{n}\right] \\
=\frac{1}{\Gamma(\alpha) \Gamma\left(\beta_{1}\right) \ldots \Gamma\left(\beta_{n}\right)} \int_{0}^{\infty} \cdots \int_{0}^{\infty} \int_{x}^{\infty} e^{-t-s_{1}-\cdots-s_{n}} t^{\alpha-1} \\
\quad \times s_{1}^{\beta_{1}-1} \cdots s_{n}^{\beta_{n}-1}{ }_{0} F_{1}\left(-; \gamma ; x_{1} s_{1} t+\cdots+x_{n} s_{n} t\right) d s_{1} \cdots d s_{n} d t \\
\quad\left(x \geqq 0 ; \Re(\alpha)>0, \Re\left(\beta_{1}\right), \ldots, \Re\left(\beta_{n}\right)>0 \text { when } x=0\right) .
\end{aligned}
$$

Proof. Using the integral representation of incomplete confluent hypergeometric function [18, p. 678, Eq.(5.2)] in the (3.3), we are led to the desired result.

In particular, for $n=2$, the corresponding integral representations for incomplete first Appell functions are presented, without proof, as asserted by Theorems 5, 6 and 7 below.

Theorem 5. The following integral representation for incomplete first Appell function $\Gamma_{1}$ in (2.7) holds true:

$$
\begin{gathered}
\Gamma_{1}\left[(\alpha, x), \beta_{1}, \beta_{2} ; \gamma ; x_{1}, x_{2}\right] \\
=\frac{1}{\Gamma(\alpha)} \int_{x}^{\infty} e^{-t} t^{\alpha-1} \Phi_{2}\left[\beta_{1}, \beta_{2} ; \gamma ; x_{1} t, x_{2} t\right] d t \\
\left(x \geqq 0 ; \max \left\{\Re\left(x_{1}\right), \Re\left(x_{2}\right)\right\}<1 ; \Re(\alpha)>0 \text { when } x=0\right) .
\end{gathered}
$$

Theorem 6. The following double integral representation for incomplete first Appell function $\Gamma_{1}$ in (2.7) holds true:

$$
\begin{gathered}
\Gamma_{1}\left[(\alpha, x), \beta_{1}, \beta_{2} ; \gamma ; x_{1}, x_{2}\right]=\frac{1}{\Gamma\left(\beta_{1}\right) \Gamma\left(\beta_{2}\right)} \int_{0}^{\infty} \int_{0}^{\infty} e^{-s_{1}-s_{2}} \\
\quad \times s_{1}^{\beta_{1}-1} s_{2}^{\beta_{2}-1}{ }_{1} \Gamma_{1}\left((\alpha, x) ; \gamma ; x_{1} s_{1}+x_{2} s_{2}\right) d s_{1} d s_{2} \\
\left(x \geqq 0 ; \Re\left(\beta_{1}\right), \Re\left(\beta_{2}\right)>0 \text { when } x=0\right) .
\end{gathered}
$$

Theorem 7. The following triple integral representation for incomplete first Appell function $\Gamma_{1}$ in (2.7) holds true:

$$
\begin{gathered}
\Gamma_{1}\left[(\alpha, x), \beta_{1}, \beta_{2} ; \gamma ; x_{1}, x_{2}\right] \\
=\frac{1}{\Gamma(\alpha) \Gamma\left(\beta_{1}\right) \Gamma\left(\beta_{2}\right)} \int_{0}^{\infty} \int_{0}^{\infty} \int_{x}^{\infty} e^{-t-s_{1}-s_{2}} t^{\alpha-1} s_{1}^{\beta_{1}-1} s_{2}^{\beta_{2}-1} \\
\quad \times{ }_{0} F_{1}\left(-; \gamma ; x_{1} s_{1} t+x_{2} s_{2} t\right) d s_{1} d s_{2} d t \\
\left(x \geqq 0 ; \Re(\alpha)>0, \Re\left(\beta_{1}\right), \Re\left(\beta_{2}\right)>0 \text { when } x=0\right) .
\end{gathered}
$$


Remark 2. The Bessel function $J_{\nu}(z)$ and the modified Bessel function $I_{\nu}(z)$ are expressible in terms of hypergeometric functions as follows (see, e.g., [15]; see also [4, 10, 11, 13, 24, 25]):

$$
J_{\nu}(z)=\frac{\left(\frac{z}{2}\right)^{\nu}}{\Gamma(\nu+1)}{ }_{0} F_{1}\left(-; \nu+1 ;-\frac{1}{4} z^{2}\right) \quad\left(\nu \in \mathbb{C} \backslash \mathbb{Z}^{-}\right)
$$

and

$$
I_{\nu}(z)=\frac{\left(\frac{z}{2}\right)^{\nu}}{\Gamma(\nu+1)}{ }_{0} F_{1}\left(-; \nu+1 ; \frac{1}{4} z^{2}\right) \quad\left(\nu \in \mathbb{C} \backslash \mathbb{Z}^{-}\right) .
$$

Now, applying the relationships (3.8) and (3.9) to (3.4) and (3.7), we can deduce certain interesting integral representations for the incomplete Lauricella's and Appell hypergeometric functions in (2.2) and (2.7) asserted by Corollaries 1 and 2 below. We give them without proof.

Corollary 1. Each of the following $n+1$-multiple integral representations holds true:

$$
\begin{aligned}
\Gamma_{D}^{(n)} & {\left[(\alpha, x), \beta_{1}, \beta_{2} ; \gamma+1 ;-x_{1}, \ldots,-x_{n}\right]=\frac{\Gamma(\gamma+1)}{\Gamma(\alpha) \Gamma\left(\beta_{1}\right) \ldots \Gamma\left(\beta_{n}\right)} } \\
& \times \int_{0}^{\infty} \cdots \int_{0}^{\infty} \int_{x}^{\infty} e^{-t-s_{1}-\cdots-s_{n}} t^{\alpha-\frac{\gamma}{2}-1} s_{1}^{\beta_{1}-1} \cdots s_{n}^{\beta_{n}-1} \\
& \times\left(x_{1} s_{1}+\cdots+x_{n} s_{n}\right)^{-\frac{\gamma}{2}} J_{\gamma}\left(2 \sqrt{x_{1} s_{1} t+\cdots+x_{n} s_{n} t}\right) d s_{1} \cdots d s_{n} d t
\end{aligned}
$$

and

$$
\begin{aligned}
\Gamma_{D}^{(n)} & {\left[(\alpha, x), \beta_{1}, \ldots, \beta_{n} ; \gamma+1 ; x_{1}, \ldots, x_{n}\right]=\frac{\Gamma(\gamma+1)}{\Gamma(\alpha) \Gamma\left(\beta_{1}\right) \ldots \Gamma\left(\beta_{n}\right)} } \\
& \times \int_{0}^{\infty} \cdots \int_{0}^{\infty} \int_{x}^{\infty} e^{-t-s_{1}-\cdots-s_{n}} t^{\alpha-\frac{\gamma}{2}-1} s_{1}^{\beta_{1}-1} \cdots s_{n}^{\beta_{n}-1} \\
& \times\left(x_{1} s_{1}+\cdots+x_{n} s_{n}\right)^{-\frac{\gamma}{2}} I_{\gamma}\left(2 \sqrt{x_{1} s_{1} t+\cdots+x_{n} s_{n} t}\right) d s_{1} \cdots d s_{n} d t
\end{aligned}
$$

provided that the integrals involved are convergent.

Corollary 2. Each of the following triple integral representations holds true:

$$
\begin{aligned}
& \Gamma_{1}\left[(\alpha, x), \beta_{1}, \beta_{2} ; \gamma+1 ;-x_{1},-x_{2}\right]=\frac{\Gamma(\gamma+1)}{\Gamma(\alpha) \Gamma\left(\beta_{1}\right) \Gamma\left(\beta_{2}\right)} \\
& \quad \times \int_{0}^{\infty} \int_{0}^{\infty} \int_{x}^{\infty} e^{-t-s_{1}-s_{2}} t^{\alpha-\frac{\gamma}{2}-1} s_{1}^{\beta_{1}-1} s_{2}^{\beta_{2}-1}\left(x_{1} s_{1}+x_{2} s_{2}\right)^{-\frac{\gamma}{2}} \\
& \quad \times J_{\gamma}\left(2 \sqrt{x_{1} s_{1} t+x_{2} s_{2} t}\right) d s_{1} d s_{2} d t
\end{aligned}
$$


and

$$
\begin{aligned}
& \Gamma_{1}\left[(\alpha, x), \beta_{1}, \beta_{2} ; \gamma+1 ; x_{1}, x_{2}\right]=\frac{\Gamma(\gamma+1)}{\Gamma(\alpha) \Gamma\left(\beta_{1}\right) \Gamma\left(\beta_{2}\right)} \\
& \quad \times \int_{0}^{\infty} \int_{0}^{\infty} \int_{x}^{\infty} e^{-t-s_{1}-s_{2}} t^{\alpha-\frac{\gamma}{2}-1} s_{1}^{\beta_{1}-1} s_{2}^{\beta_{2}-1}\left(x_{1} s_{1}+x_{2} s_{2}\right)^{-\frac{\gamma}{2}} \\
& \quad \times I_{\gamma}\left(2 \sqrt{x_{1} s_{1} t+x_{2} s_{2} t}\right) d s_{1} d s_{2} d t
\end{aligned}
$$

provided that the integrals involved are convergent.

\section{Derivative formula}

Differentiating both sides of $(2.2)$ with respect to $x_{1}, \ldots, x_{n}$ partially $m_{1}, \ldots, m_{n}$ times, respectively, we obtain a derivative formula for the incomplete Lauricella's hypergeometric function $\Gamma_{D}^{(n)}$ given in the following theorem.

Theorem 8. The following derivative formula for $\Gamma_{D}^{(n)}$ holds true:

$$
\begin{aligned}
& \frac{\partial^{m_{1}+\cdots+m_{n}}}{\partial x_{1}^{m_{1}} \ldots \partial x_{n}^{m_{n}}} \Gamma_{D}^{(n)}\left[(\alpha, x), \beta_{1}, \ldots, \beta_{n} ; \gamma ; x_{1}, \ldots, x_{n}\right] \\
& \quad=\frac{(\alpha)_{m_{1}+\ldots+m_{n}}\left(\beta_{1}\right)_{m_{1}} \ldots\left(\beta_{n}\right)_{m_{n}}}{(\gamma)_{m_{1}+\ldots+m_{n}}} \Gamma_{D}^{(n)}\left[\left(\alpha+m_{1}+\cdots+m_{n}, x\right),\right. \\
& \left.\quad \beta_{1}+m_{1}, \ldots, \beta_{n}+m_{n} ; \gamma+m_{1}+\ldots+m_{n} ; x_{1}, \ldots, x_{n}\right] .
\end{aligned}
$$

It is noted that a similar argument will establish the corresponding derivative formula of incomplete first Appell function $\Gamma_{1}$ as a special case of (4.1) when $n=2$.

\section{Recurrence Relation}

In this section, we present a recurrence relation for incomplete Lauricella functions.

Theorem 9. The following recurrence relations for $\Gamma_{D}^{(n)}$ holds true: 


$$
\begin{aligned}
& \Gamma_{D}^{(n)}\left[(\alpha, x), \beta_{1}, \ldots, \beta_{n} ; \gamma ; x_{1}, \ldots, x_{n}\right] \\
& =\Gamma_{D}^{(n)}\left[(\alpha, x), \beta_{1}, \ldots, \beta_{n} ; \gamma-1 ; x_{1}, \ldots, x_{n}\right] \\
& \quad+\frac{\alpha \beta_{1} x_{1}}{\gamma(1-\gamma)} \Gamma_{D}^{(n)}\left[(\alpha+1, x), \beta_{1}+1, \beta_{2} \ldots, \beta_{n} ; \gamma+1 ; x_{1}, \ldots, x_{n}\right] \ldots \\
& \quad+\frac{\alpha \beta_{n} x_{n}}{\gamma(1-\gamma)} \Gamma_{D}^{(n)}\left[(\alpha+1, x), \beta_{1}, \beta_{2} \ldots, \beta_{n-1}, \beta_{n}+1 ; \gamma+1 ; x_{1}, \ldots, x_{n}\right] .
\end{aligned}
$$

Proof. Using the well-known contiguous relation for the function ${ }_{0} F_{1}$ (see $[16$, p. 12])

$$
{ }_{0} F_{1}(-; \gamma-1 ; x)-{ }_{0} F_{1}(-; \gamma ; x)-\frac{x}{\gamma(\gamma-1)}{ }_{0} F_{1}(-; \gamma+1 ; x)=0
$$

in the integral representation (3.4), we are led to the desired result.

It is noted that a similar argument will establish the corresponding recurrence relation of incomplete first Appell function $\Gamma_{1}$ as a special case of (5.1) when $n=2$.

Acknowledgements. The authors would like to express their deep gratitude for the reviewers's helpful comments to improve this paper in the present form.

\section{References}

[1] M. Abramowitz, I. A. Stegun (Eds.), Handbook of Mathematical Functions with Formulas, Graphs, and Mathematical Tables, Tenth Printing, National Bureau of Standards, Applied Mathematics Series 55, Washington, DC, 1972; Reprinted by Dover Publications, New York, 1965.

[2] L. C. Andrews, Special Functions for Engineers and Applied Mathematicians, Macmillan Company, New York, 1984.

[3] W. N. Bailey, Generalized Hypergeometric Series, Cambridge University Press, Cambridge, 1935; Reprinted by Stechert Hafner, New York, 1964.

[4] B. C. Carlson, Special Functions of Applied Mathematics, Academic Press, New York, San Francisco and London, 1977.

[5] A. Çetinkaya, The incomplete second Appell hypergeometric functions, Appl. Math. Comput. 219 (2013), 8332-8337.

[6] M. A. Chaudhry and S. M. Zubair, On a Class of Incomplete Gamma Functions with Applications, Chapman and Hall, (CRC Press Company), Boca Raton, London, New York and Washington, D. C., 2001.

[7] A. Erdélyi, W. Magnus, F. Oberhettinger and F. G. Tricomi, Higher Transcendental Functions, Vol. I, McGraw-Hill Book Company, New York, Toronto and London, 1953.

[8] A. Erdélyi, W. Magnus, F. Oberhettinger and F. G. Tricomi, Higher Transcendental Functions, Vol. II, McGraw-Hill Book Company, New York, Toronto and London, 1953. 
[9] A. A. Kilbas, H. M. Srivastava and J.J. Trujillo, Theory and Applications of Fractional Differential Equations, North-Holland Mathematical Studies, 204, Elsevier (North-Holland) Science Publishers, Amsterdam, London and New York, 2006.

[10] Y. L. Luke, Mathematical Functions and Their Approximations, Academic Press, New York, San Francisco and London, 1975.

[11] W. Magnus, F. Oberhettinger and R. P. Soni, Formulas and Theorems for the Special Functions of Mathematical Physics, Third Enlarged edition, Die Grundlehren der Mathematischen Wissenschaften in Einzeldarstellungen mit besonderer Berücksichtingung der Anwendungsgebiete, Bd. 52, Springer-Verlag, Berlin, Heidelberg and New York, 1966.

[12] K. B. Oldham, J. Myland and J. Spanier, An Atlas of Functions, With Equator, The Atlas Function Calculator, Second edi. [With 1 CD-ROM (Windows)], Springer, Berlin, Heidelberg and New York, 2009.

[13] F. W. J. Olver, D. W. Lozier, R. F. Boisvert and C. W. Clark (Eds.), NIST Handbook of Mathematical Functions [With 1 CD-ROM (Windows, Macintosh and UNIX)], US Department of Commerce, National Institute of Standards and Technology, Washington, DC, 2010; Cambridge University Press, Cambridge, London and New York, 2010.

[14] A. P. Prudnikov, Yu. A. Brychkov and O. I. Marichev, Integrals and Series, Vol. II, Gordon and Breach Science Publishers, New York, 1990.

[15] E. D. Rainville, Special Functions, Macmillan Company, New York, 1960; Reprinted by Chelsea Publishing Company, Bronx, New York, 1971.

[16] L. J. Slater, Confluent Hypergeometric Functions, Cambridge University Press, Cambridge, London, and New York, 1960.

[17] L. J. Slater, Generalized Hypergeometric Functions, Cambridge University Press, Cambridge, London, and New York, 1966.

[18] H. M. Srivastava, M. A. Chaudhry and R. P. Agarwal, The incomplete Pochhammer symbols and their applications to hypergeometric and related functions, Integral Transforms Spec. Funct. 23 (2012), 659-683.

[19] H. M. Srivastava and J. Choi, Series Associated with the Zeta and Related Functions, Kluwer, Acedemic Publishers, Dordrecht, Boston and London, 2001.

[20] H. M. Srivastava and J. Choi, Zeta and q-Zeta Functions and Associated Series and Integrals, Elsevier Science, Publishers, Amsterdam, London and New York, 2012 .

[21] H. M. Srivastava and P. W. Karlsson, Multiple Gaussian Hypergeometric Series, Halsted Press, (Ellis Horwood Limited, Chichester), John Wiley and Sons, New York, Chichester, Brisbane and Toronto, 1985.

[22] H. M. Srivastava and H. L. Manocha, A Treatise on Generating Functions, Halsted Press, (Ellis Horwood Limited, Chichester), John Wiley and Sons, New York, Chichester, Brisbane and Toronto, 1984.

[23] N. M. Temme, Special Functions: An Introduction to Classical Functions of Mathematical Physics, John Wiley and Sons, New York, Chichester, Brisbane and Toronto, 1996.

[24] G. N. Watson, A Treatise on the Theory of Bessel Functions, Second edition, Cambridge University Press, Cambridge, London and New York, 1944. 
[25] E. T. Whittaker and G. N. Watson, A Course of Modern Analysis: An Introduction to the General Theory of Infinite Processes and of Analytic Functions; With an Account of the Principal Transcendental Functions, Fourth edition, Cambridge University Press, Cambridge, London and New York, 1963.

Junesang Choi

Department of Mathematics, Dongguk University, Gyeongju 780-714, Republic of Korea.

E-mail: junesang@mail.dongguk.ac.kr

Rakesh K. Parmar

Department of Mathematics, Government College of Engineering and Technology,

Bikaner-334004, Rajasthan State, India.

E-mail: rakeshparmar27@gmail.com

Purnima Chopra

Department of Mathematics, Marudhar Engineering College,

Bikaner-334004, Rajasthan State, India.

E-mail: purnimachopra05@gmail.com 